PAPER

\title{
Magnetic resonance brain imaging in patients with acute stroke: feasibility and patient related difficulties
}

\author{
P J Hand, J M Wardlaw, A M Rowat, J A Haisma, R I Lindley, M S Dennis
}

See end of article for authors' affiliations

J Neurol Neurosurg Psychiatry 2005;76:1525-1527. doi: 10.1136/jnnp.2005.062539

Correspondence to: Professor J M Wardlaw, Division of Clinical Neurosciences, University of Edinburgh, Western General Hospital, Crewe Road, Edinburgh EH4 $2 \mathrm{XU}, \mathrm{UK}$; joanna. wardlaw@ed.ac.uk

Received 4 January 2005 Revised version received 9 March 2005

Accepted 17 March 2005

\begin{abstract}
Objectives: To assess organisational and patient specific limitations and safety of magnetic resonance imaging (MRI) as the first line investigation for hospital admitted stroke patients.

Methods: Consecutive patients admitted with acute stroke were assessed and an attempt was made to perform MRI in all patients. Oxygen saturation and interventions required during scanning were recorded. Results: Among 136 patients recruited over 34 weeks, 85 (62\%) underwent MRI. The patients' medical instability (15 of the 53 not scanned), contraindications to MRI (six of the 53 not scanned), and rapid symptom resolution ( 10 of the 53 not scanned) were the main reasons for not performing MRI. Of the 85 patients who underwent MRI, 26 required physical intervention, 17 did not complete scanning, and 11 of the 61 who had successful oxygen saturation monitoring were hypoxic during MRI. Organisational limitations accounted for only $13 \%$ of failures to scan.

Conclusions: Up to $85 \%$ of hospital admitted acute stroke patients could have MRI as first line imaging investigation, but medical instability is the major limitation. Hypoxia is frequent in MRI. Patients should be monitored carefully, possibly by an experienced clinician, during scanning.
\end{abstract}

M agnetic resonance imaging (MRI) has been advocated as a method of improving the selection of patients with stroke for thrombolysis. ${ }^{1}$ However, MRI has more contraindications, is more difficult to perform in ill patients, and takes longer than computed tomography scanning.

The current issue is to determine the proportion of patients with acute stroke in whom MRI is achievable, and how to identify (in the acute situation) those in whom MRI is unlikely to be successful. Studies of imaging appearances in selected patients, ${ }^{2}$ or which were retrospective but gave no indication of the actual number of suspected strokes not referred for MRI in the same time period, ${ }^{3}{ }^{4}$ have not provided such information. One recent prospective study found that $20 \%$ of 144 stroke patients recruited "at the hospital door" could not have MRI (of whom half had MRI contraindications and half were medically unstable). ${ }^{5}$ No study has investigated whether MRI is safe in acute stroke.

We aimed to determine: (1) whether it was possible to use MRI as the first line assessment for all suspected acute strokes, and (2) the limitations and safety of MRI in acute stroke.

\section{METHODS}

From among all consecutive admissions to our hospital (population served, 500 000) with suspected acute stroke, patients were eligible for the MRI feasibility study if they could be assessed by a clinical research fellow and scanned within 24 hours of stroke, and the clinical diagnosis was probable or definite stroke (transient ischaemic attacks and obvious non-strokes were excluded).

We recorded time of stroke and assessment, clinical features, neurological deficit (National Institutes of Health Stroke Scale Score (NIHSS): http://www.strokecenter.org/ trials/scales/) and stroke subtype. ${ }^{6}$ An experienced neurologist assessed the risk of complications occurring during MRI. We noted whether $x$ rays were required to exclude metal.

All reasons for not performing MRI were recorded. Patients were scanned on a 1.5 T GE Signa Echoplanar MRI scanner, providing virtually unrestricted access for stroke during normal working hours, with: gradient echo, diffusion weighted imaging (three orthogonal gradients), perfusion imaging (dynamic bolus tracking with $20 \mathrm{ml}$ gadolinium), and T2 axial images (12 minutes of imaging time).

During MRI, we attempted to recorded pulse and arterial oxygen saturation $\left(\mathrm{SaO}_{2}\right)$ every five minutes $\left({ }^{\mathrm{TM}}\right.$ Odam Maglife MR compatible monitor with continuous photoplethysmographic signal every eight seconds). Hypoxia (fall in $\mathrm{SaO}_{2}$ to $90 \%$ or less, lasting at least one minute), interventions during scanning (medical-for example, administering a drug; nursing-for example, suction, oxygen, other; or physical reassurance-for example, hand squeeze, not just verbal), and premature termination of scanning, were recorded.

Data were analysed by means of non-parametric tests using Microsoft Excel (version 97 SR-2; ${ }^{\circ}$ Microsoft Corporation, 1997) and SPSS for Windows (version 10.0.5; ${ }^{\odot}$ SPSS Inc, 1999).

\section{RESULTS}

Among all patients admitted with suspected stroke $(\mathrm{n}=336)$ in nine months, 138 presentations by 136 patients were recruited to the MRI feasibility study (two patients presented twice). The major reasons for non-recruitment from all suspected stroke $(\mathrm{n}=336)$ to the MRI study $(\mathrm{n}=136)$ were (1) patient arrived too late or the research fellow was busy with other patients (not included: median, 26 hours; interquartile range, 13-51; included: median, eight hours; interquartile range, 3-19), or (2) clinical diagnosis not definite or probable stroke $(n=116)$. Other patient characteristics for the MRI study sample were very similar to all admitted patients with stroke.

Of 138 MRI study eligible patients, 53 (38\%) could not be scanned (fig 1). Patients not scanned were significantly older (median age, $77 v 74$ years; oldest patient, $97 v$ 99; $\mathrm{p}=0.03$ ), had more severe strokes (median NIHSS $11 \vee 5$; $=0.03$ ), and were more often drowsy (57\% v 32\%; $\mathrm{p}=0.007)$ than those who were scanned.

Abbreviations: MRI, magnetic resonance imaging; NIHSS, National Institutes of Health Stroke Scale Score; $\mathrm{SaO}_{2}$, arterial oxygen saturation 


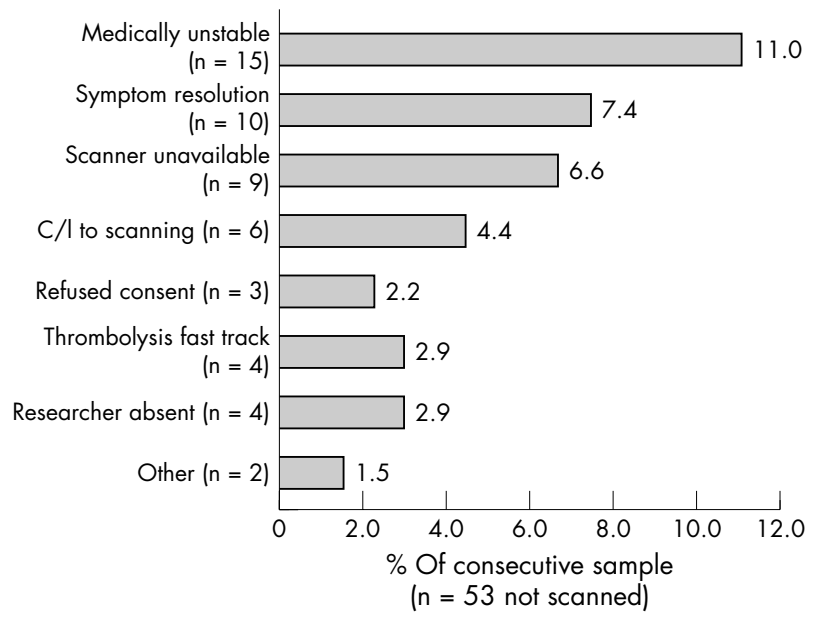

Figure 1 Reasons for failure to carry out a magnetic resonance imaging scan on eligible patients. C/I, contraindications.

Patient related factors were the most common reason for not scanning (fig 1): 15 of 53 (11.0\% of the whole MRI study). These factors included being too confused to lie flat and cooperate, low Glasgow Coma Score, acute pulmonary oedema with oxygen saturation of $60 \%$ despite oxygen treatment, rapid symptom resolution (transient ischaemic attack), MRI contraindications (pacemaker, four; recent surgery with metal sutures, one; prosthetic mitral valve of uncertain type, one), and claustrophobia (three). Organisational factors affected 14 patients: scanner nonavailability (9, outside working hours or service days) and fast tracked to computed tomography for thrombolysis because the wait for MRI was too long (four). Orbit $x$ rays to exclude ocular metal added 20-30 minutes of delay in 13\% of patients (all normal).

Twenty four patients could not be monitored in MRI (table 1): confused or aphasic patients had difficulty retaining the finger probe, or the signal was weak (irregular pulse, poor tissue perfusion). Non-monitored patients were non-significantly more likely to have a higher NIHSS, be confused, drowsy, or have atrial fibrillation.

Eleven of the 61 patients monitored became hypoxic during MRI (lowest recorded saturation during scanning, $74 \%$ ). All patients had similar oxygen saturation on admission and at the start of scanning (hypoxic in MRI $v$ not hypoxic: admission $96.5 \% \vee 97 \%$ and start of MRI 94\% v 96\%; both $\mathrm{p}=\mathrm{NS}$ ). Patients who became hypoxic were slightly older, but of similar stroke severity to those without hypoxia.
Although seven patients received oxygen during scanning, in only two was $\mathrm{SaO}_{2}$ monitored successfully.

Seventeen of the 85 patients terminated the scanning prematurely (10 claustrophobia; four stopped because the patient was too unwell). Total median time in MRI was 25 minutes, including 10 minutes (interquartile range, 7-17) of "settling time".

Twenty seven patients needed physical reassurance (21) or nursing intervention (six), including two started on oxygen while undergoing MRI. Patients requiring intervention had more severe strokes (median NIHSS $8 v 4.5 ; \mathrm{p}<0.01$ ), were aphasic $\left(44 \%\right.$ v 22\%; $\left.\mathrm{p}=0.04 ; \chi^{2}\right)$, older, more confused or drowsy, and were far more likely to terminate the scan prematurely $\left(56 \% \quad v 3 \% ; \mathrm{p}<0.01 ; \chi^{2}\right)$ than those not requiring intervention.

The patients judged to be at high risk $(n=15)$ of complications in scanning more often needed intervention during scanning, or terminated the scan prematurely $(\mathrm{p}<0.01$ for both) than those judged to be at low risk $(\mathrm{n}=70)$. However, hypoxia was twice as frequent in low compared with high risk patients $(20 \% v 10 \% ; \mathrm{p}=\mathrm{NS})$.

\section{DISCUSSION}

It is important to improve the use of MRI through better awareness of potential problems and patient specific practical limitations. We investigated the question "if MRI were to be the first line investigation for all patients with suspected stroke, what proportion could be scanned, what difficulties would be encountered, and is it safe?". Of 138 consecutive patients with acute stroke, we were only able to start MRI scanning in $85(62 \%)$. With more clinicians and 24 hour scanner availability (organisational factors) we could have scanned up to $85 \%$; that is, $13 \%$ more patients. However, the major reason for not scanning was the patients' medical instability. A shorter scanning protocol could improve tolerance, but most of the median 25 minutes of "scanning time" was not image acquisition, but settling/reassuring/ managing the patient.

Is MRI in acute stroke safe? The observers in the MRI room frequently detected hypoxia and patient distress, not apparent from the control room. Patients with severe strokes, reduced conscious level, or coexisting medical problems who had greatest need of monitoring were most difficult to monitor. Those patients who would become hypoxic (11 of the 61 patients who we could monitor) could not be predicted beforehand. Those judged to be at "low risk" of difficulties during scanning were twice as likely to become hypoxic than those at "high risk", and worryingly, there may have been even more hypoxic episodes among those with more severe strokes whom we could not monitor.

Table 1 Features of those patients undergoing MRI $(n=85)$ : patients who had $\mathrm{SaO}_{2}$ monitoring versus those in whom monitoring was not possible

\begin{tabular}{|c|c|c|c|c|c|}
\hline \multirow[b]{2}{*}{ Feature } & \multicolumn{2}{|c|}{$\mathrm{SaO}_{2}$ recordings possible $(n=61)$} & \multicolumn{2}{|c|}{ No $\mathrm{SaO}_{2}$ recordings possible $(\mathrm{n}=24$ ) } & \multirow[b]{2}{*}{ p Value* } \\
\hline & Number & Range or $\%$ & Number & Range or $\%$ & \\
\hline Median age (IQR) & 73 years & $61-82$ & 77 years & $71-81$ & 0.441 \\
\hline Irregular pulse & 13 & $22 \%$ & 6 & $26 \%$ & 0.725 \\
\hline Confusion & 10 & $24 \%$ & 5 & $36 \%$ & 0.411 \\
\hline Decreased conscious level & 11 & $18 \%$ & 5 & $21 \%$ & 0.766 \\
\hline Aphasia & 18 & $30 \%$ & 7 & $30 \%$ & 0.975 \\
\hline Median NIHSS (IQR) & 5.0 & $3-9$ & 6.0 & $4-9$ & 0.356 \\
\hline TACS & 9 & $15 \%$ & 3 & $13 \%$ & 0.788 \\
\hline Median scan time (IQR) & $27 \mathrm{~min}$ & $20-42$ & $21 \mathrm{~min}$ & $16-30$ & 0.026 \\
\hline Median $\mathrm{SaO}_{2}$ in ARU (IQR) & $97 \%$ & $95-98$ & $96 \%$ & $95-98$ & 0.542 \\
\hline
\end{tabular}

*Determined by Mann-Whitney $U$ test (for continuous variables) and $\chi^{2}$ test (for categorical variables)

$\mathrm{ARU}$, acute respiratory unit; IQR, interquartile range; MRI, magnetic resonance imaging; NIHSS, National Institutes of Health Stroke Scale Score; SaO 2 , arterial oxygen saturation; TACS, total anterior circulation syndrome. 
Few previous studies gave details of patient inclusions and exclusions, ${ }^{7}$ and none provided patient tolerability or safety data. Small sample sizes-for example, 64 patients over two years from 1200 patients admitted each year $1<3 \%$ were scanned), ${ }^{2}$ or 66 of 347 patients scanned over a five year period ${ }^{8}$ suggest similar difficulties. The only other study prospectively recruiting "at the hospital door" had very similar results to ours: $20 \%$ of 144 patients could not undergo MRI because of MRI contraindications (half) or medical factors (half). ${ }^{5}$ More of our patients were claustrophobic (9.4\%; 95\% confidence interval, $5.6 \%$ to $15.5 \%$ ) than in other, less acute stroke studies. ${ }^{9-12}$

In conclusion, there are potential risks of early MRI. Researchers seeing younger, more medically stable patients with stroke may be less aware of this. Because stroke predominantly affects the elderly, and the average age is rising, ${ }^{13}$ it is important to assess imaging in a representative sample. Lying supine for up to 25 minutes increases the risk of aspiration, yet patient observation is difficult, and monitoring may not work in those at greatest risk of complications. In acute stroke, nearly $20 \%$ of patients may desaturate to $90 \% \mathrm{SaO}_{2}$ or less, 33\% may require some intervention, and $20 \%$ may not complete the full MRI sequence. Hypoxia could not be predicted. Physicians managing acute stroke need to be aware of this, particularly as MRI availability for stroke increases. It would be unfortunate if, in striving for greater diagnostic certainty with MRI, physicians inadvertently lost valuable time in hyper-acute stroke through lack of awareness of these practical limitations and increased the risk of penumbral damage from hypoxia. Trained personnel should be with the patient during scanning. Further research should investigate the balance of information gained versus the safety and practicality of MRI.

\section{ACKNOWLEDGEMENTS}

Funding by the Scottish Executive Chief Scientist's Office (reference number CZB/4/14); the Health Foundation (reference number 2268/ 1114); the SHEFC Brain Imaging Research Centre for Scotland (www.dcn.ed.ac.uk/bic).

\section{Authors' affiliations}

J M Wardlaw, A M Rowat, M S Dennis, Division of Clinical Neurosciences, Western General Hospital, Crewe Road, Edinburgh EH4 2XU, UK
P J Hand, RMH Stroke Centre, Department of Neurology, Royal Melbourne Hospital, Victoria, Australia 3050

J A Haisma, Department of Rehabilitation Medicine, Erasmus Medical Centre, Postbus 2040, 3000 CA Rotterdam, The Netherlands

R I Lindley, Department of Geriatric Medicine, Westmead Hospital, Western Clinical School (C24), University of Sydney, Westmead, NSW 2006, Australia

Competing interests: none declared

Ethics and consent: The study was approved by the Lothian research ethics committee reference number LREC/1702/98/4/1, the regional committee for single centre research conducted in Lothian Region. Consent to participate in the study was obtained from the patient, or assent from a relative, as approved by the ethics committee.

\section{REFERENCES}

1 Hacke W, Warach S. Diffusion-weighted MRI as an evolving standard of care in acute stroke. Neurology 2000;54:1548-9

2 Schellinger PD, Jansen O, Fiebach JB, et al. Feasibility and practicality of MR imaging of stroke in the management of hyperacute cerebral ischemia. AJNR Am J Neuroradiol, 2000;21:1184-9.

3 Mullins ME, Schaefer PW, Sorensen AG, et al. CT and conventional and diffusion-weighted MR imaging in acute stroke: study in 691 patients at presentation to the emergency department. Neuroradiology 2002;224:353-60.

4 Buckley BT, Wainwright A, Meagher T, et al. Audit of a policy of magnetic resonance imaging with diffusion-weighted imaging as first-line neuroimaging for in-patients with clinically suspected acute stroke. Clin Radiol 2003;58:234-7.

5 Singer OC, Sitzer M, du Mesnil de Rochemont R, et al. Practical limitations of acute stroke MRI due to patient-related problems. Neurology 2004;62:1848-9.

6 Bamford J, Sandercock P, Dennis M, et al. Classification and natural history of clinically identifiable subtypes of cerebral infarction. Lancet 1991;337:1521-6.

7 Keir SL, Wardlaw J. Systematic review of diffusion and perfusion imaging in acute ischaemic stroke. Stroke 2000;31:2731.

8 Baird AE, Dambrosia J, Janket S, et al. A three-item scale for the early prediction of stroke recovery. Lancet 2001;357:2095-9.

9 Flaherty JA, Hoskinson K. Emotional distress during magnetic resonance imaging. N Engl J Med 1989;320:467-8.

10 Dantendorfer K, Wimberger D, Katschnig H, et al. Claustrophobia in MRI scanners. Lancet 1991;338:761-2.

11 Avrahami E. Panic attacks during MR imaging: treatment with i.v. diazepam. AJNR Am J Neuroradiol 1990;11:833-5.

12 Sarii SA, Abdullah BJ, Kumar GTAH, et al. Failed magnetic resonance imaging examinations due to claustrophobia. Australas Radiol 1998;42:293-5.

13 Rothwell PM, Coull AJ, Giles MF, et al. Change in stroke incidence, mortality, case-fatality, severity, and risk factors in Oxfordshire, UK from 1981 to 2004 (Oxford vascular study). Lancet 2004;363:1925-33. 